\title{
Some New Double-Sequence Spaces in 2-Normed Spaces Defined by Ideal Convergence and an Orlicz Function
}

\section{Orhan Tŭ̆, Mutlay Doğan, and Abdullah Kurudirek}

\author{
Ishik University, Arbil, Iraq \\ Correspondence should be addressed to Orhan Tŭ̆, tugorhan27@gmail.com
}

Received 14 December 2011; Accepted 26 January 2012

Academic Editors: S. Anita and S. Liu

Copyright (C) 2012 Orhan Tuğ et al. This is an open access article distributed under the Creative Commons Attribution License, which permits unrestricted use, distribution, and reproduction in any medium, provided the original work is properly cited.

We generalize some sequence spaces from single to double, we study some topological properties of these double sequence spaces by using ideal convergence, difference sequence spaces, and an Orlicz function in 2-normed spaces, and we give some results related to these sequence spaces.

\section{Introduction}

The concept of $I$-convergent was introduced by Das et al. [1] and developed by many scholars. B. Tripathy and B. C. Tripathy [2] extended the concept of $I$-convergence from single sequence to double sequence. Some difference double-sequence spaces and paranormed double-sequence spaces defined by Orlicz function were introduced by Tripathy and Sarma $[3,4]$. And also some new single- and double-sequence spaces defined in 2-normed spaces using ideal convergence and an Orlicz function were introduced by Savaş [5, 6].

Recall that an Orlicz Function, which was presented by Karasnoselskii and Rutishky [7] $M:[0, \infty) \rightarrow[0, \infty)$ is continuous, convex, nondecreasing function such that $M(0)=0$ and $M(x)>0$ for $x>0$ and $M(x) \rightarrow \infty$ as $x \rightarrow \infty$.

An Orlicz function $M$ can be represented in the following integral form: $M(x)=$ $\int_{0}^{x} p(t) d t$, where $p$ is the known kernel of $M$, right differential for $t \geq 0, p(0)=0, p(t)>0$ for $t>0, p$ is nondecreasing, and $p(t) \rightarrow \infty$ as $t \rightarrow \infty$.

Ruckle [8] and Maddox [9] presented and discussed that if convexity of Orlicz function $M$ is replaced by $M(x+y) \leq M(x)+M(y)$ then this function is called Modulus function. 
An Orlicz function $M$ is said to satisfy $\Delta_{2}$-condition for all values of $u$, if there exists constant $K>0$, such that $M(2 u) \leq K M(u),(u \geq 0)$. The $\Delta_{2}$-condition is equivalent to the inequality $M(L u) \leq K L M(u)$ for all values of $u$ and for $L>1$ being satisfied [7].

The notion of difference double-sequence spaces was introduced by Tripathy and Sarma [3]. These notions are further studied by Tripathy and Sarma [4]. Let $\left(a_{n k}\right)$ be a double sequence. Then the operator $\Delta$ is defined as $\Delta a_{n k}=a_{n, k}-a_{n, k+1}-a_{n+1, k}+a_{n+1, k+1}$, for all $k, n \in \mathbb{N}$.

Let us recall some well-known concepts that $(X,\|\cdot\|)$ is a normed space and a sequence $\left(x_{m n}\right)_{(m, n \in \mathbb{N})}$ of elements of $X$ is called to be statistically convergent to $x \in X$ if the set $A(\varepsilon)=$ $\left\{m, n \in \mathbb{N}:\left\|x_{m n}-x\right\| \geq \varepsilon\right\}$ has zero natural density for each $\varepsilon>0[2]$.

A real double-sequence $x=\left(x_{k, l}\right)$ is said to be statistically bounded above if there exists a real number $D>0$ such that $\delta\left\{k: x_{k, l}>D\right\}=0$. A real double-sequence $x=\left(x_{k, l}\right)$ is said to be statistically bounded below if there exists a real number $E>0$ such that $\delta\left\{k: x_{k, l}<E\right\}=0$. If a real double-sequence $x=\left(x_{k, l}\right)$ is statistically bounded both above and below, then we say that $x=\left(x_{k, l}\right)$ is statistically bounded. It is clear that any bounded double-sequence is also statistically bounded [10].

A family $I \subset 2^{Y}$ of subset of a nonempty set $Y$ is said to be an ideal in $Y$ if

(i) $\emptyset \in Y$,

(ii) $A, B \in I$ imply $A \cup B \in I$,

(iii) $A \in I, B \subset A$ imply $B \in I[11,12]$.

$I \subset 2^{Y}$ is called an admissible ideal in $Y$ if and only if it contains $\{\{y\}: y \in Y\}$ [13].

Let $I \subset 2^{\mathbb{N} \times \mathbb{N}}$ be a nontrivial ideal (i.e., $I \neq \emptyset$ and $\mathbb{N} \times \mathbb{N} \notin I$ ) in $\mathrm{N}$. The sequence $\left(x_{m n}\right)_{(m, n \in \mathbb{N})}$ is said to be $I$-convergent to $x \in X$ if for each $\varepsilon>0$ the set $A(\varepsilon)=\{m, n \in$ $\left.\mathbb{N}:\left\|x_{m n}-x\right\| \geq \varepsilon\right\} \in I[1]$.

A real double-sequence $x=\left(x_{k, l}\right)$ is said to be $I$-bounded above if there exists a real number $G>0$ such that $\left\{k: x_{k, l}>G\right\} \in I$. A real double-sequence $x=\left(x_{k, l}\right)$ is said to be $I$-bounded below if there exists a real number $H>0$ such that $\left\{k: x_{k, l}<H\right\} \in I$. If a real double-sequence $x=\left(x_{k, l}\right)$ is $I$-bounded both above and below, then we say that $x=\left(x_{k, l}\right)$ is $I$-bounded. One can observe easily that any bounded double sequence is I-bounded [14].

Let $X$ be a real vector space of dimension $d$, where $2 \leq d<\infty$. A 2-norm on $X$ is a function $\|\cdot, \cdot\|: X \times X \rightarrow \mathbb{R}$ which satisfied the following four conditions:

(i) $\|x, y\|=0$ if and only if $x$ and $y$ are linear dependent,

(ii) $\|x, y\|=\|y, x\|$,

(iii) $\|\alpha x, y\|=|\alpha|\|x, y\|, \alpha \in R$,

(iv) $\|x, y+z\| \leq\|x, y\|+\|x, z\|$, the pair $(X,\|\cdot, \cdot\|)$ is then called a 2-normed space $[15,16]$.

The sequence $\left(x_{k}\right)_{k \in \mathbb{N}}$ in a 2-normed space $(X,\|\cdot, \cdot\|)$ is said to be convergent to $L$ in $X$ if $\lim _{k \rightarrow \infty}\left\|x_{k}-L, z\right\|=0$ for every $z \in X$. In this instance, we write $\lim _{k \rightarrow \infty}\left\|x_{k}, z\right\|=\|L, z\|$ [17].

Let $I \subset 2^{\mathbb{N}}$ be a nontrivial ideal in $\mathbb{N}$. The sequence $\left(x_{k}\right)_{k \in \mathbb{N}}$ in a 2-normed space $X$ is said to be $I$-convergent to $x$, if for every $\varepsilon>0$ and $z$ in $X$ the set $A(\varepsilon)=\left\{k \in \mathbb{N}:\left\|x_{k}-x, z\right\| \geq \varepsilon\right\}$ belongs to $I$ [18].

The following inequalities which will be used throughout the paper can be introduced like in Maddox [19]. If $0 \leq p_{k, l} \leq \sup _{k, l} p_{k, l}=H, D=\max \left(1,2^{H-1}\right)$ then $\left|a_{k, l}+b_{k, l}\right|^{p_{k, l}} \leq$ $D\left\{\left|a_{k, l}\right|^{p_{k, l}}+\left|b_{k, l}\right|^{p_{k, l}}\right\}$ for all $k, l$ and $a_{k, l}, b_{k, l} \in \mathbb{C}$. And also $|a|^{p_{k, l}} \leq \max \left(1,2^{H}\right)$ for all $a \in \mathbb{C}$. 


\section{Main Results}

Let $\lambda=\left(\lambda_{n}\right)$ and $\mu=\left(\mu_{m}\right)$ be two nondecreasing sequences of positive real numbers both of which tend to $\infty$ as $n$ and $m$ approach $\infty$, respectively. Also let $\lambda_{n+1} \leq \lambda_{n}+1, \lambda_{1}=0$ and $\mu_{m+1} \leq \mu_{m}+1, \mu_{1}=0$. Let $I$ be an admissible ideal of $\mathbb{N} \times \mathbb{N}, M$ an Orlicz function, and $(X,\|\cdot, \cdot\|)$ a 2-normed spaces. In addition let $p=p_{k, l}$ be a bounded sequence of positive real numbers. With $S^{\prime \prime}(2-X)$, we symbolize the space of all double sequences defined over $(X,\|\cdot, \cdot\|)$. Now we define the following double sequence spaces:

$$
\begin{aligned}
& \left(W^{I}\right)_{2}(\lambda, M, \Delta, p,\|\cdot, \cdot\|) \\
& =\left\{x \in S^{\prime \prime}(2-X): \forall \varepsilon>0\left\{m, n \in \mathbb{N}: \frac{1}{\bar{\lambda}_{n, m}} \sum_{k, l \in I_{n, m}}\left[M\left(\left\|\frac{\Delta x_{k, l}-L}{\rho}, z\right\|\right)\right]^{p_{k, l}} \geq \varepsilon\right\} \in I\right. \\
& \text { for some } \rho>0, L \in X \text { and each } z \in X\} \text {, } \\
& \left(W_{0}^{I}\right)_{2}(\lambda, M, \Delta, p,\|\cdot, \cdot\|) \\
& =\left\{x \in S^{\prime \prime}(2-X): \forall \varepsilon>0\left\{m, n \in \mathbb{N}: \frac{1}{\bar{\lambda}_{n, m}} \sum_{k, l \in I_{n, m}}\left[M\left(\left\|\frac{\Delta x_{k, l}}{\rho}, z\right\|\right)\right]^{p_{k, l}} \geq \varepsilon\right\} \in I\right. \\
& \text { for some } \rho>0 \text { and each } z \in X\} \text {, } \\
& \left(W_{\infty}\right)_{2}(\lambda, M, \Delta, p,\|\cdot, \cdot\|) \\
& =\left\{x \in S^{\prime \prime}(2-X): \exists K>0 \text { s.t. } \sup _{m, n} \frac{1}{\bar{\lambda}_{n, m}} \sum_{k, l \in I_{n, m}}\left[M\left(\left\|\frac{\Delta x_{k, l}}{\rho}, z\right\|\right)\right]^{p_{k, l}} \leq K\right. \\
& \text { for some } \rho>0 \text { and each } z \in X\} \text {, } \\
& \left(W_{\infty}^{I}\right)_{2}(\lambda, M, \Delta, p,\|\cdot, \cdot\|) \\
& =\left\{x \in S^{\prime \prime}(2-X): \exists K>0 \text { s.t. }\left\{m, n \in \mathbb{N}: \frac{1}{\bar{\lambda}_{n, m}} \sum_{k, l \in I_{n, m}}\left[M\left(\left\|\frac{\Delta x_{k, l}}{\rho}, z\right\|\right)\right]^{p_{k, l}} \geq K\right\} \in I\right. \\
& \text { for some } \rho>0 \text { and each } z \in X\} \text {, }
\end{aligned}
$$

where $I_{n}=\left[n-\lambda_{n}+1, n\right], I_{m}=\left[m-\mu_{m}+1, m\right]$, and $\bar{\lambda}_{n, m}=\lambda_{n} \mu_{m}$. Throughout this paper we will denote $\lambda_{n} \mu_{m}$ by $\bar{\lambda}_{n, m}$ and $\left(k \in I_{n}, l \in I_{m}\right)$ by $(k, l) \in \bar{I}_{n, m}$. 
Theorem 2.1. $\left(W^{I}\right)_{2}(\lambda, M, \Delta, p,\|\cdot, \cdot\|),\left(W_{0}^{I}\right)_{2}(\lambda, M, \Delta, p,\|\cdot, \cdot\|),\left(W_{\infty}^{I}\right)_{2}(\lambda, M, \Delta, p,\|\cdot, \cdot\|)$ are linear spaces.

Proof. Because all the statements can be proved in a similar way, we will prove the assertion for $\left(W_{0}^{I}\right)_{2}(\lambda, M, \Delta, p,\|\cdot, \cdot\|)$. Suppose that $x, y \in\left(W_{0}^{I}\right)_{2}(\lambda, M, \Delta, p,\|\cdot, \cdot\|)$ and $\alpha, \beta \in \mathbb{R}$. So

$$
\left.\begin{array}{c}
\left\{m, n \in \mathbb{N}: \frac{1}{\bar{\lambda}_{n, m}} \sum_{k, l \in I_{n, m}}\left[M\left(\left\|\frac{\Delta x_{k, l}}{\rho_{1}}, z\right\|\right)\right]^{p_{k, l}} \geq \varepsilon\right\} \in I, \\
\text { for some } \rho_{1}>0
\end{array}\right\}
$$

Since we study $(X,\|\cdot, \cdot\|)$ 2-normed, $\Delta$ is linear and $M$ is an Orlicz function, we have the following inequality:

$$
\begin{aligned}
\frac{1}{\bar{\lambda}_{n, m}} \sum_{k, l \in I_{n, m}}\left[M\left(\left\|\frac{\Delta\left(\alpha x_{k, l}+\beta y_{k, l}\right)}{\left(|\alpha| \rho_{1}+|\beta| \rho_{2}\right)}, z\right\|\right)\right]^{p_{k, l}} \\
\leq D \frac{1}{\bar{\lambda}_{n, m}} \sum_{k, l \in I_{n, m}}\left[\frac{|\alpha|}{\left(|\alpha| \rho_{1}+|\beta| \rho_{2}\right)} M\left(\left\|\frac{\Delta x_{k, l}}{\rho_{1}}, z\right\|\right)\right]^{p_{k, l}} \\
\quad+D \frac{1}{\bar{\lambda}_{n, m}} \sum_{k, l \in I_{n, m}}\left[\frac{|\beta|}{\left(|\alpha| \rho_{1}+|\beta| \rho_{2}\right)} M\left(\left\|\frac{\Delta y_{k, l}}{\rho_{2}}, z\right\|\right)\right]^{p_{k, l}} \\
\leq D F \frac{1}{\bar{\lambda}_{n, m}} \sum_{k, l \in I_{n, m}}\left[M\left(\left\|\frac{\Delta x_{k, l}}{\rho_{1}}, z\right\|\right)\right]^{p_{k, l}} \\
\quad+D F \frac{1}{\bar{\lambda}_{n, m}} \sum_{k, l \in I_{n, m}}\left[M\left(\left\|\frac{\Delta y_{k, l}}{\rho_{2}}, z\right\|\right)\right]^{p_{k, l}}
\end{aligned}
$$

where $F=\max \left[1,\left(|\alpha| /\left(|\alpha| \rho_{1}+|\beta| \rho_{2}\right)\right)^{H},\left(|\beta| /\left(|\alpha| \rho_{1}+|\beta| \rho_{2}\right)\right)^{H}\right]$.

From the above inequality we get

$$
\begin{array}{r}
\left\{m, n \in \mathbb{N}: \frac{1}{\bar{\lambda}_{n, m}} \sum_{k, l \in I_{n, m}}\left[M\left(\left\|\frac{\Delta\left(\alpha x_{k, l}+\beta y_{k, l}\right)}{\left(|\alpha| \rho_{1}+|\beta| \rho_{2}\right)}, z\right\|\right)\right]^{p_{k, l}} \geq \varepsilon\right\} \\
\subseteq\left\{m, n \in \mathbb{N}: D F \frac{1}{\bar{\lambda}_{n, m}} \sum_{k, l \in I_{n, m}}\left[M\left(\left\|\frac{\Delta x_{k, l}}{\rho_{1}}, z\right\|\right)\right]^{p_{k, l}} \geq \frac{\varepsilon}{2}\right\} \\
\cup\left\{m, n \in \mathbb{N}: D F \frac{1}{\bar{\lambda}_{n, m}} \sum_{k, l \in I_{n, m}}\left[M\left(\left\|\frac{\Delta y_{k, l}}{\rho_{2}}, z\right\|\right)\right]^{p_{k, l}} \geq \frac{\varepsilon}{2}\right\}
\end{array}
$$

the two set on the right side belonging to $I$ and this completes the proof. 
Theorem 2.2. For any fixed $m, n \in \mathbb{N},\left(W_{\infty}\right)_{2}(\lambda, M, \Delta, p,\|\cdot, \cdot\|)$ is a paranormed space with the paranorm defined by

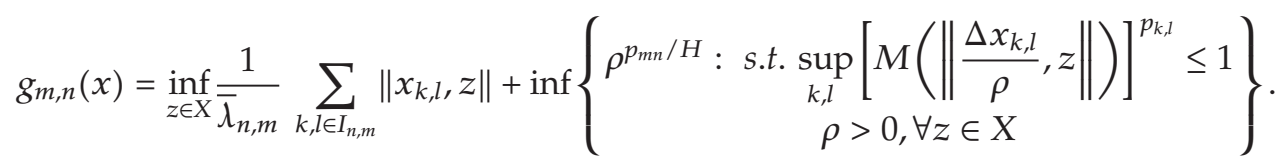

Proof. (i) $g_{m, n}(0)=0$ and (ii) $g_{m, n}(-x)=g_{m, n}(x)$ are easy to prove. So we leave them out. (iii) Let us take $x, y \in\left(W_{\infty}\right)_{2}(\lambda, M, \Delta, p,\|\cdot, \cdot\|)$. Let

$$
\begin{aligned}
& A(x)=\left\{\rho>0: \sup _{k, l}\left[M\left(\left\|\frac{\Delta x_{k, l}}{\rho}, z\right\|\right)\right]^{p_{k, l}} \leq 1, \forall z \in X\right\} \\
& A(y)=\left\{\rho>0: \sup _{k, l}\left[M\left(\left\|\frac{\Delta y_{k, l}}{\rho}, z\right\|\right)\right]^{p_{k, l}} \leq 1, \forall z \in X\right\} .
\end{aligned}
$$

Let $\rho_{1} \in A(x)$ and $\rho_{2} \in A(y)$. Then if $\rho=\rho_{1}+\rho_{2}$ then we have

$$
M\left(\left\|\frac{\Delta\left(x_{k, l}+y_{k, l}\right)}{\rho}, z\right\|\right) \leq \frac{\rho_{1}}{\rho_{1}+\rho_{2}} M\left(\left\|\frac{\Delta x_{k, l}}{\rho_{1}}, z\right\|\right)+\frac{\rho_{2}}{\rho_{1}+\rho_{2}} M\left(\left\|\frac{\Delta y_{k, l}}{\rho_{2}}, z\right\|\right) .
$$

Thus

$$
\begin{aligned}
\sup _{k, l} M\left(\left\|\frac{\Delta\left(x_{k, l}+y_{k, l}\right)}{\rho_{1}+\rho_{2}}, z\right\|\right) \leq 1 \\
g_{m, n}(x+y)=\inf _{z \in X} \frac{1}{\bar{\lambda}_{n, m}} \sum_{k, l \in I_{n, m}}\left\|x_{k, l}+y_{k, l}, z\right\|+\inf \left\{\left(\rho_{1}+\rho_{2}\right)^{p_{m n} / H}: \rho_{1} \in A(x), \rho_{2} \in A(y)\right\} \\
\leq \inf _{z \in X} \frac{1}{\bar{\lambda}_{n, m}} \sum_{k, l \in I_{n, m}}\left\|x_{k, l}, z\right\|+\inf \left\{\left(\rho_{1}\right)^{p_{m n} / H}: \rho_{1} \in A(x)\right\} \\
\quad+\inf _{z \in X} \frac{1}{\bar{\lambda}_{n, m}} \sum_{k, l \in I_{n, m}}\left\|y_{k, l}, z\right\|+\inf \left\{\left(\rho_{2}\right)^{p_{m n} / H}: \rho_{2} \in A(y)\right\} \\
=g_{m, n}(x)+g_{m, n}(y) .
\end{aligned}
$$


(iv) Let $\sigma^{i} \rightarrow \sigma$, where $\sigma, \sigma^{i} \in \mathbb{C}$ and $g_{m, n}\left(x_{k, l}^{i}-x\right) \rightarrow 0$ as $i \rightarrow \infty$. We have to show that $g_{m, n}\left(\sigma^{i} x_{k, l}^{i}-\sigma x\right) \rightarrow 0$ as $i \rightarrow \infty$. Let

$$
\begin{gathered}
A\left(x^{i}\right) \quad=\left\{\rho_{i}>0: \sup _{k, l}\left[M\left(\left\|\frac{\Delta x_{k, l}^{i}}{\rho_{i}}, z\right\|\right)\right]^{p_{k, l}} \leq 1, \forall z \in X\right\}, \\
A\left(x^{i}-x\right)=\left\{\rho_{i}^{\prime}>0: \sup _{k, l}\left[M\left(\left\|\frac{\left(\Delta x_{k, l}^{i}-x_{k, l}\right)}{\rho_{i}^{\prime}}, z\right\|\right)\right]^{p_{k, l}} \leq 1, \forall z \in X\right\} .
\end{gathered}
$$

If $\rho_{i} \in A\left(x^{i}\right)$ and $\rho_{i}^{\prime} \in A\left(x^{i}-x\right)$ then we observe that

$$
\begin{aligned}
M\left(\left\|\frac{\Delta\left(\sigma^{i} x_{k, l}^{i}-\sigma x_{k, l}\right)}{\rho_{i}\left|\sigma^{i}-\sigma\right|+\rho_{i}^{\prime}|\sigma|}, z\right\|\right) & M\left(\left\|\frac{\Delta\left(\sigma^{i} x_{k, l}^{i}-\sigma x_{k, l}^{i}\right)}{\rho_{i}\left|\sigma^{i}-\sigma\right|+\rho_{i}^{\prime}|\sigma|}, z\right\|+\left\|\frac{\Delta\left(\sigma x_{k, l}^{i}-\sigma x_{k, l}^{i}\right)}{\rho_{i}\left|\sigma^{i}-\sigma\right|+\rho_{i}^{\prime}|\sigma|}, z\right\|\right) \\
\leq & \frac{\rho_{i}\left|\sigma^{i}-\sigma\right|}{\rho_{i}\left|\sigma^{i}-\sigma\right|+\rho_{i}^{\prime}|\sigma|} M\left(\left\|\frac{\Delta x_{k, l}^{i}}{\rho_{i}}, z\right\|\right) \\
& +\frac{\rho_{i}^{\prime}|\sigma|}{\rho_{i}\left|\sigma^{i}-\sigma\right|+\rho_{i}^{\prime}|\sigma|} M\left(\left\|\frac{\Delta\left(x_{k, l}^{i}-x_{k, l}\right)}{\rho_{i}^{\prime}}, z\right\|\right) .
\end{aligned}
$$

From above inequality it obviously follows that

$$
\left(M\left(\left\|\frac{\Delta\left(\sigma^{i} x_{k, l}^{i}-\sigma x_{k, l}\right)}{\rho_{i}\left|\sigma^{i}-\sigma\right|+\rho_{i}^{\prime}|\sigma|}, z\right\|\right)\right)^{p_{k, l}} \leq 1
$$

and consequently

$$
\begin{aligned}
& g_{m, n}\left(\sigma^{i} x^{i}-\sigma x\right) \\
& \leq \inf _{z \in \mathrm{X}} \frac{1}{\bar{\lambda}_{n, m}} \sum_{k, l \in I_{n, m}}\left\|\sigma^{i} x_{k, l}^{i}-\sigma x_{k, l}, z\right\| \\
& \quad+\inf \left\{\left(\rho_{i}\left|\sigma^{i}-\sigma\right|+\rho_{i}^{\prime}|\sigma|\right)^{p_{m n} / H}: \rho_{i} \in A\left(x^{i}\right), \rho_{i}^{\prime} \in A\left(x^{i}-x\right)\right\} \\
& \leq\left|\sigma^{i}-\sigma\right| \inf _{z \in X} \frac{1}{\bar{\lambda}_{n, m}} \sum_{k, l \in I_{n, m}}\left\|x_{k, l}^{i}, z\right\|+|\sigma| \inf _{z \in X} \frac{1}{\bar{\lambda}_{n, m}} \sum_{k, l \in I_{n, m}}\left\|x_{k, l}^{i}-x_{k, l}, z\right\| \\
& \quad+\left(\left|\sigma^{i}-\sigma\right|\right)^{p_{m n} / H} \inf _{\left\{\left(\rho_{i}\right)^{p_{m n} / H}: \rho_{i} \in A\left(x^{i}\right)\right\}}
\end{aligned}
$$


ISRN Mathematical Analysis

$$
\begin{aligned}
& +(|\sigma|)^{p_{m n} / H} \inf \left\{\left(\rho_{i}^{\prime}\right)^{p_{m n} / H}: \rho_{i}^{\prime} \in A\left(x^{i}-x\right)\right\} \\
\leq & \max \left\{\left|\sigma^{i}-\sigma\right|,\left(\left|\sigma^{i}-\sigma\right|\right)^{p_{m n} / H}\right\} g_{m, n}\left(x^{i}\right)+\max \left\{|\sigma|,(|\sigma|)^{p_{m n} / H}\right\} g_{m, n}\left(x^{i}-x\right) .
\end{aligned}
$$

Note that $g_{m, n}\left(x^{i}\right)<g_{m, n}(x)+g_{m, n}\left(x^{i}-x\right)$ for all $i \in \mathbb{N}$. Hence by our assumption the right-hand side tends to 0 as $i \rightarrow \infty$ and the results follow. This completes the proof of the theorem.

Theorem 2.3. Let $M$ be an Orlicz function which satisfies the $\Delta_{2}$-condition. Then $\left(W_{0}^{I}\right)_{2}(\lambda, M, \Delta, p,\|\cdot, \cdot\|) \subset\left(W^{I}\right)_{2}(\lambda, M, \Delta, p,\|\cdot, \cdot\|) \subset\left(W_{\infty}^{I}\right)_{2}(\lambda, M, \Delta, p,\|\cdot, \cdot\|)$ and the inclusions are strict.

Proof. Because it can be proven in a similar way, we introduce the proof for $\left(W^{I}\right)_{2}(\lambda, M, \Delta, p,\|\cdot, \cdot\|) \subset\left(W_{\infty}^{I}\right)_{2}(\lambda, M, \Delta, p,\|\cdot, \cdot\|)$ only. Let $x \in\left(W^{I}\right)_{2}(\lambda, M, \Delta, p,\|\cdot, \cdot\|)$. Then

$$
\begin{aligned}
\frac{1}{\bar{\lambda}_{n, m}} \sum_{k, l \in I_{n, m}}\left[M\left(\left\|\frac{\Delta x_{k, l}}{2 \rho}, z\right\|\right)\right]^{p_{k, l}} & =\frac{1}{\bar{\lambda}_{n, m}} \sum_{k, l \in I_{n, m}}\left[M\left(\left\|\frac{\Delta x_{k, l}+L-L}{2 \rho}, z\right\|\right)\right]^{p_{k, l}} \\
\leq & \frac{1}{\bar{\lambda}_{n, m}} \sum_{k, l \in I_{n, m}}\left[M\left(\left\|\frac{\Delta x_{k, l}-L}{2 \rho}, z\right\|+\left\|\frac{L}{2 \rho}, z\right\|\right)\right]^{p_{k, l}} \\
\leq & D G \frac{1}{\bar{\lambda}_{n, m}} \sum_{k, l \in I_{n, m}}\left[M\left(\left\|\frac{\Delta x_{k, l}-L}{\rho}, z\right\|\right)\right]^{p_{k, l}} \\
& +D G_{\bar{\lambda}_{n, m}} \sum_{k, l \in I_{n, m}}\left[M\left(\left\|\frac{L}{\rho}, z\right\|\right)\right]^{p_{k, l}}
\end{aligned}
$$

where $G=\max \left\{1,(1 / 2)^{H}\right\}$ and we get from $\Delta_{2}$-condition

$$
D G \frac{1}{\bar{\lambda}_{n, m}} \sum_{k, l \in I_{n, m}}\left(\left[M\left(\left\|\frac{\Delta x_{k, l}-L}{\rho}, z\right\|\right)\right]^{p_{k, l}}+\left[\frac{K}{\rho \delta}\|L, z\| M(2)\right]^{H}\right)
$$

so $x \in\left(W_{\infty}^{I}\right)_{2}(\lambda, M, \Delta, p,\|\cdot, \cdot\|)$ and this completes the proof.

Theorem 2.4. Let $X(\Delta)$ stand for $\left(W^{I}\right)_{2}(\lambda, M, \Delta, p,\|\cdot, \cdot\|),\left(W_{0}^{I}\right)_{2}(\lambda, M, \Delta, p,\|\cdot, \cdot\|)$ or $\left(W_{\infty}^{I}\right)_{2}(\lambda, M, \Delta, p,\|\cdot, \cdot\|)$. Then the inclusion $X(\Delta) \subset X\left(\Delta^{2}\right)$ is strict.

Proof. Because this can be proven in a similar way, we will give the proof for $\left(W_{0}^{I}\right)_{2}(\lambda, M, \Delta, p,\|\cdot, \cdot\|)$ only. Let $x \in\left(W_{0}^{I}\right)_{2}(\lambda, M, \Delta, p,\|\cdot, \cdot\|)$. Then given $\varepsilon>0$ we have

$$
\left\{m, n \in \mathbb{N}: \frac{1}{\bar{\lambda}_{n, m}} \sum_{k, l \in I_{n, m}}\left[M\left(\left\|\frac{\Delta x_{k, l}}{\rho}, z\right\|\right)\right]^{p_{k, l}} \geq \varepsilon\right\} \in I .
$$


Since $M$ is nondecreasing and convex it follows that

$$
\begin{aligned}
\frac{1}{\bar{\lambda}_{n, m}} & \sum_{k, l \in I_{n, m}}\left[M\left(\left\|\frac{\Delta^{2} x_{k, l}}{4 \rho}, z\right\|\right)\right]^{p_{k, l}} \\
= & \frac{1}{\bar{\lambda}_{n, m}} \sum_{k, l \in I_{n, m}}\left[M\left(\left\|\frac{\Delta x_{k, l}-\Delta x_{k+1, l}-\Delta x_{k, l+1}+\Delta x_{k+1, l+1}}{4 \rho}, z\right\|\right)\right]^{p_{k, l}} \\
\leq & \frac{1}{\bar{\lambda}_{n, m}} \sum_{k, l \in I_{n, m}}\left[M\left(\left\|\frac{\Delta x_{k, l}-\Delta x_{k+1, l}}{4 \rho}, z\right\|+\left\|\frac{\Delta x_{k+1, l+1}-\Delta x_{k, l+1}}{4 \rho}, z\right\|\right)\right]^{p_{k, l}} \\
\leq & D G \frac{1}{\bar{\lambda}_{n, m}} \sum_{k, l \in I_{n, m}}\left[M\left(\left\|\frac{\Delta x_{k, l}-\Delta x_{k+1, l}}{2 \rho}, z\right\|\right)\right]^{p_{k, l}} \\
& +D G \frac{1}{\bar{\lambda}_{n, m}} \sum_{k, l \in I_{n, m}}\left[M\left(\left\|\frac{\Delta x_{k+1, l+1}-\Delta x_{k, l+1}}{2 \rho}, z\right\|\right)\right]^{p_{k, l}},
\end{aligned}
$$

where $G=\max \left\{1,(1 / 2)^{H}\right\}$. Similarly from the convexity and nondecreasing properties of $M$

$$
\begin{aligned}
D G & \frac{1}{\bar{\lambda}_{n, m}} \sum_{k, l \in I_{n, m}}\left(\left[M\left(\left\|\frac{\Delta x_{k, l}-\Delta x_{k+1, l}}{2 \rho}, z\right\|\right)\right]^{p_{k, l}}+\left[M\left(\left\|\frac{\Delta x_{k+1, l+1}-\Delta x_{k, l+1}}{2 \rho}, z\right\|\right)\right]^{p_{k, l}}\right) \\
\leq & D G \frac{1}{\bar{\lambda}_{n, m}} \sum_{k, l \in I_{n, m}}\left[M\left(\left\|\frac{\Delta x_{k, l}}{2 \rho}, z\right\|+\left\|\frac{\Delta x_{k+1, l}}{2 \rho}, z\right\|\right)\right]^{p_{k, l}} \\
& +D G \frac{1}{\bar{\lambda}_{n, m}} \sum_{k, l \in I_{n, m}}\left[M\left(\left\|\frac{\Delta x_{k+1, l+1}}{2 \rho}, z\right\|+\left\|\frac{\Delta x_{k, l+1}}{2 \rho}, z\right\|\right)\right]^{p_{k, l}} \\
\leq & D^{2} G^{2} \frac{1}{\bar{\lambda}_{n, m}} \sum_{k, l \in I_{n, m}}\left(\left[M\left(\left\|\frac{\Delta x_{k, l} l}{\rho}, z\right\|\right)\right]^{p_{k, l}}+\left[M\left(\left\|\frac{\Delta x_{k+1, l}}{\rho}, z\right\|\right)\right]^{p_{k, l}}\right) \\
& +D^{2} G^{2} \frac{1}{\bar{\lambda}_{n, m}} \sum_{k, l \in I_{n, m}}\left(\left[M\left(\left\|\frac{\Delta x_{k, l+1}}{\rho}, z\right\|\right)\right]^{p_{k, l}}+\left[M\left(\left\|\frac{\Delta x_{k+1, l+1}}{\rho}, z\right\|\right)\right]^{p_{k, l}}\right), \\
m, n & \left.\in \mathbb{N}: \frac{1}{\bar{\lambda}_{n, m}} \sum_{k, l \in I_{n, m}}\left[M\left(\left\|\frac{\Delta^{2} x_{k, l}}{\rho}, z\right\|\right)\right]^{p_{k, l}} \geq \varepsilon\right\} \\
\leq & \left\{m, n \in \mathbb{N}: D^{2} G^{2} \frac{1}{\bar{\lambda}_{n, m}} \sum_{k, l \in I_{n, m}}\left[M\left(\left\|\frac{\Delta x_{k, l}}{\rho}, z\right\|\right)\right]^{p_{k, l}} \geq \frac{\varepsilon}{4}\right\} \\
& \cup\left\{m, n \in \mathbb{N}: D^{2} G^{2} \frac{1}{\bar{\lambda}_{n, m}} \sum_{k, l \in I_{n, m}}\left[M\left(\left\|\frac{\Delta x_{k+1, l}}{\rho}, z\right\|\right)\right]^{p_{k, l}} \geq \frac{\varepsilon}{4}\right\}
\end{aligned}
$$




$$
\begin{aligned}
& \cup\left\{m, n \in \mathbb{N}: D^{2} G^{2} \frac{1}{\bar{\lambda}_{n, m}} \sum_{k, l \in I_{n, m}}\left[M\left(\left\|\frac{\Delta x_{k, l+1}}{\rho}, z\right\|\right)\right]^{p_{k, l}} \geq \frac{\varepsilon}{4}\right\} \\
& \left.\cup\left\{m, n \in \mathbb{N}: D^{2} G^{2} \frac{1}{\bar{\lambda}_{n, m}} \sum_{k, l \in I_{n, m}}\left[M\left(\left\|\frac{\Delta x_{k+1, l+1}}{\rho}, z\right\|\right)\right]^{p_{k, l}} \geq \frac{\varepsilon}{4}\right\} .\right]
\end{aligned}
$$

Since the right-hand side belongs to I so does the left hand side.

Theorem 2.5. Let $M, M_{1}, M_{2}$ be Orlicz function. Then one has

(i) $\left(W_{0}^{I}\right)_{2}\left(\lambda, M_{1}, \Delta, p,\|\cdot, \cdot\|\right) \subseteq\left(W_{0}^{I}\right)_{2}\left(\lambda, M_{1} M_{1}, \Delta, p,\|\cdot, \cdot\|\right)$ provided $\left(p_{k, l}\right)$ is such that $H_{0}=\inf p_{k, l}>0$,

(ii) $\left(W_{0}^{I}\right)_{2}\left(\lambda, M_{1}, \Delta, p,\|\cdot, \cdot\|\right) \cap\left(W_{0}^{I}\right)_{2}\left(\lambda, M_{2}, \Delta, p,\|\cdot, \cdot\|\right) \subseteq\left(W_{0}^{I}\right)_{2}\left(\lambda, M_{1}+M_{2}, \Delta, p,\|\cdot, \cdot\|\right)$.

Proof. (i) For given $\varepsilon>0$, we can choose $\varepsilon_{0}>0$ such that $\max \left\{\varepsilon_{0}^{H}, \varepsilon_{0}^{H_{0}}\right\}<\varepsilon$. Because of the continuity of $M$, we can choose $0<\delta<1$ such that $0<t<\delta \Rightarrow M(t)<\varepsilon_{0}$. Let $x \in$ $\left(W_{0}^{I}\right)_{2}\left(\lambda, M_{1}, \Delta, p,\|\cdot, \cdot\|\right)$. We can write from the definition

$$
A(\delta)=\left\{m, n \in \mathbb{N}: \frac{1}{\bar{\lambda}_{n, m}} \sum_{k, l \in I_{n, m}}\left[M_{1}\left(\left\|\frac{\Delta x_{k, l}}{\rho}, z\right\|\right)\right]^{p_{k, l}} \geq \delta^{H}\right\} \in I .
$$

Hence if $m, n \notin A(\delta)$, then

$$
\frac{1}{\bar{\lambda}_{n, m}} \sum_{k, l \in I_{n, m}}\left[M_{1}\left(\left\|\frac{\Delta x_{k, l}}{\rho}, z\right\|\right)\right]^{p_{k, l}}<\delta^{H},
$$

that is,

$$
\sum_{k, l \in I_{n, m}}\left[M_{1}\left(\left\|\frac{\Delta x_{k, l}}{\rho}, z\right\|\right)\right]^{p_{k, l}}<\bar{\lambda}_{n, m} \delta^{H},
$$

that is,

$$
\left[M_{1}\left(\left\|\frac{\Delta x_{k, l}}{\rho}, z\right\|\right)\right]^{p_{k, l}}<\delta^{H}, \quad \forall k, l \in I_{n, m},
$$

that is,

$$
M_{1}\left(\left\|\frac{\Delta x_{k, l}}{\rho}, z\right\|\right)<\delta, \quad \forall k, l \in I_{n, m} .
$$


From this inequality using continuity of $M$ we must have

$$
M\left(M_{1}\left(\left\|\frac{\Delta x_{k, l}}{\rho}, z\right\|\right)\right)<\varepsilon_{0}, \quad \forall k, l \in I_{n, m},
$$

which consequently implies that

$$
\sum_{k, l \in I_{n, m}}\left[M\left(M_{1}\left(\left\|\frac{\Delta x_{k, l}}{\rho}, z\right\|\right)\right)\right]^{p_{k, l}}<\bar{\lambda}_{n, m} \max \left\{\varepsilon_{0}^{H}, \varepsilon_{0}^{H_{0}}\right\}<\bar{\lambda}_{n, m} \varepsilon,
$$

that is,

$$
\frac{1}{\bar{\lambda}_{n, m}} \sum_{k, l \in I_{n, m}}\left[M\left(M_{1}\left(\left\|\frac{\Delta x_{k, l}}{\rho}, z\right\|\right)\right)\right]^{p_{k, l}}<\varepsilon
$$

This show that

$$
\left\{m, n \in \mathbb{N}: \frac{1}{\bar{\lambda}_{n, m}} \sum_{k, l \in I_{n, m}}\left[M\left(M_{1}\left(\left\|\frac{\Delta x_{k, l}}{\rho}, z\right\|\right)\right)\right]^{p_{k, l}} \geq \varepsilon\right\} \subset A(\delta),
$$

and from the definition ideal the left side belongs to $I$. This proves the result.

(ii) Let $\left(x_{k, l}\right) \in\left(W_{0}^{I}\right)_{2}\left(\lambda, M_{1}, \Delta, p,\|\cdot, \cdot\|\right) \cap\left(W_{0}^{I}\right)_{2}\left(\lambda, M_{2}, \Delta, p,\|\cdot, \cdot\|\right)$. Then the fact

$$
\left[\left(M_{1}+M_{2}\right)\left(\left\|\frac{\Delta x_{k, l}}{\rho}, z\right\|\right)\right]^{p_{k, l}} \leq D\left(\left[M_{1}\left(\left\|\frac{\Delta x_{k, l}}{\rho}, z\right\|\right)\right]^{p_{k, l}}+\left[M_{2}\left(\left\|\frac{\Delta x_{k, l}}{\rho}, z\right\|\right)\right]^{p_{k, l}}\right)
$$

give us the result. Thus $\left(x_{k, l}\right) \in\left(W_{0}^{I}\right)_{2}\left(\lambda, M_{1}+M_{2}, \Delta, p,\|\cdot, \cdot\|\right)$, hence the proof.

Definition 2.6. Let $X$ be sequence space. Then $X$ is called solid if $\left(\alpha_{k} x_{k}\right) \in X$ whenever $\left(x_{k}\right) \in$ $X$ for all sequence $\left(\alpha_{k}\right)$ of scalar with $\left|\alpha_{k}\right| \leq 1$ for all $k \in \mathbb{N}$.

Theorem 2.7. The sequence spaces $\left(W_{0}^{I}\right)_{2}(\lambda, M, \Delta, p,\|\cdot, \cdot\|),\left(W_{\infty}^{I}\right)_{2}(\lambda, M, \Delta, p,\|\cdot, \cdot\|)$ are solid.

Proof. We give the proof for $\left(W_{0}^{I}\right)_{2}(\lambda, M, \Delta, p,\|\cdot, \cdot\|)$ only. Let $\left(x_{k, l}\right) \in\left(W_{0}^{I}\right)_{2}(\lambda, M, \Delta, p,\|\cdot, \cdot\|)$ and $\left(\alpha_{k, l}\right)$ be a sequence of scalars such that $\left|\alpha_{k, l}\right| \leq 1$ for all $k, l \in \mathbb{N}$. Then we have

$$
\begin{aligned}
& \left\{m, n \in \mathbb{N}: \frac{1}{\bar{\lambda}_{n, m}} \sum_{k, l \in I_{n, m}}\left[M\left(\left\|\frac{\Delta\left(\alpha_{k, l} x_{k, l}\right)}{\rho}, z\right\|\right)\right]^{p_{k, l}} \geq \varepsilon\right\} \\
& \subseteq\left\{m, n \in \mathbb{N}: \frac{C}{\bar{\lambda}_{n, m}} \sum_{k, l \in I_{n, m}}\left[M\left(\left\|\frac{\Delta x_{k, l}}{\rho}, \mathrm{z}\right\|\right)\right]^{p_{k, l}} \geq \varepsilon\right\} \in I,
\end{aligned}
$$

where $C=\max \left\{1,\left(\left|\alpha_{k, l}\right|\right)^{H}\right\}$. Hence $\left(\alpha_{k, l} x_{k, l}\right) \in\left(W_{0}^{I}\right)_{2}(\lambda, M, \Delta, p,\|\cdot, \cdot\|)$ for all sequences of scalars $\left(\alpha_{k, l}\right)$ with $\left|\alpha_{k, l}\right| \leq 1$ for all $k, l \in \mathbb{N}$ whenever $\left(x_{k, l}\right) \in\left(W_{0}^{I}\right)_{2}(\lambda, M, \Delta, p,\|\cdot, \cdot\|)$. 


\section{References}

[1] P. Das, P. Kostyrko, W. Wilczyński, and P. Malik, "I and $I^{*}$-convergence of double sequences," Mathematica Slovaca, vol. 58, no. 5, pp. 605-620, 2008.

[2] B. Tripathy and B. C. Tripathy, “On I-convergent double sequences,” Soochow Journal of Mathematics, vol. 31, no. 4, pp. 549-560, 2005.

[3] B. C. Tripathy and B. Sarma, "On some classes of difference double sequence spaces," Fasciculi Mathematici, vol. 41, pp. 135-142, 2009.

[4] B. C. Tripathy and B. Sarma, "Some paranormed difference double sequence spaces defined by Orlicz function," Fasciculi Mathematici, vol. 39, pp. 113-124, 2008.

[5] E. Savaş, "Some new double sequence spaces defined by Orlicz function in $n$-normed space," Journal of Inequalities and Applications, vol. 2011, Article ID 592840, 9 pages, 2011.

[6] E. Savas, " $\Delta^{m}$-strongly summable sequences spaces in 2-normed spaces defined by ideal convergence and an Orlicz function," Applied Mathematics and Computation, vol. 217, no. 1, pp. 271-276, 2010.

[7] M. A. Karasnoselskii and Y. B. Rutisky, Convex Function and Orlicz Spaces, P. Noordhoff, Groningen, Netherlands, 1961.

[8] W. H. Ruckle, "FK-spaces in which the sequence of coordinate vectors is bounded," Canadian Journal of Mathematics, vol. 25, pp. 973-978, 1973.

[9] I. J. Maddox, "Sequence spaces defined by a modulus," Mathematical Proceedings of the Cambridge Philosophical Society, vol. 100, no. 1, pp. 161-166, 1986.

[10] C. Çakan and B. Altay, "Statistically boundedness and statistical core of double sequences," Journal of Mathematical Analysis and Applications, vol. 317, no. 2, pp. 690-697, 2006.

[11] J. L. Kelley, General Topology, Springer, New York, NY, USA, 1955.

[12] C. Kuratowski, Topology I, PWN, Warszawa, Poland, 1958.

[13] P. Kostyrko, T. Šalát, and W. Wilczyński, "I-convergence," Real Analysis Exchange, vol. 26, no. 2, pp. 669-685, 2000-2001.

[14] V. Kumar and N. Singh, "I-core of double sequences," International Journal of Contemporary Mathematical Sciences, vol. 2, no. 21-24, pp. 1137-1145, 2007.

[15] S. Gähler, "2-metrische Räume und ihre topologische Struktur,” Mathematische Nachrichten, vol. 26, pp. 115-148, 1963.

[16] S. Gahler, "Lineare 2-normietre Raume," Mathematische Nachrichten, vol. 28, pp. 335-347, 1964.

[17] S. Gahler, “Unterschungen uber verallgemeinerte m-metrische Raume. II," Mathematische Nachrichten, vol. 40, pp. 229-264, 1969.

[18] A. Şahiner, M. Gürdal, S. Saltan, and H. Gunawan, "Ideal convergence in 2-normed spaces," Taiwanese Journal of Mathematics, vol. 11, no. 5, pp. 1477-1484, 2007.

[19] I. J. Maddox, Elements of Functional Analysis, Cambridge University Press, London, UK, 1970. 


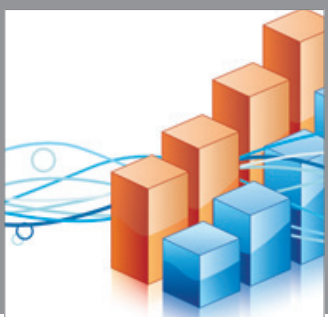

Advances in

Operations Research

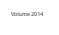

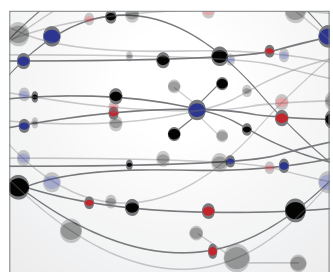

\section{The Scientific} World Journal
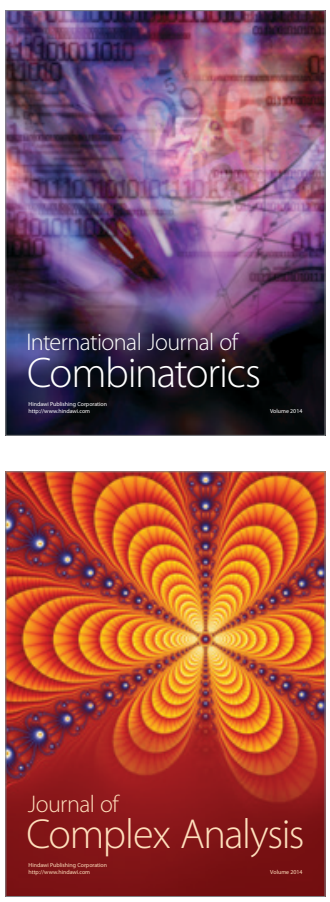

International Journal of

Mathematics and

Mathematical

Sciences
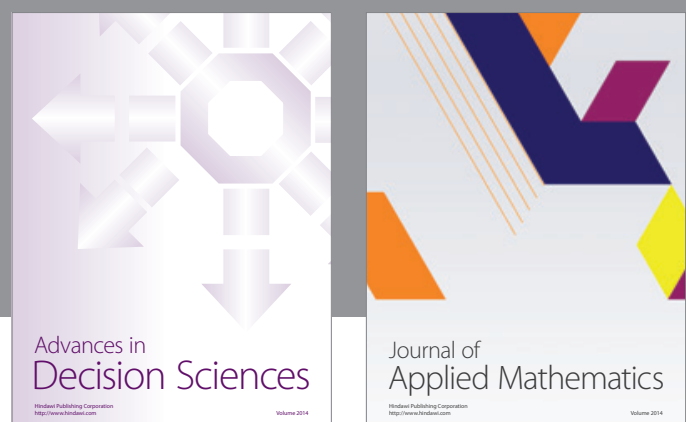

Journal of

Applied Mathematics
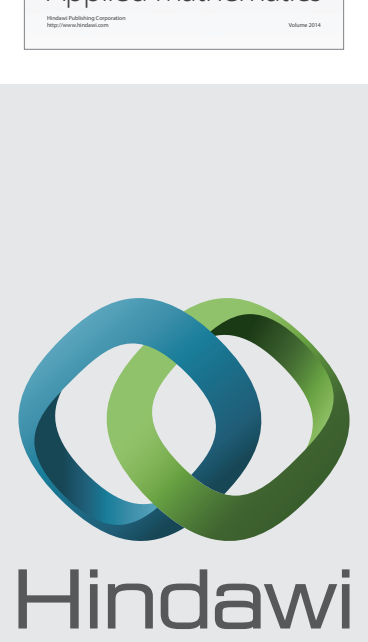

Submit your manuscripts at http://www.hindawi.com
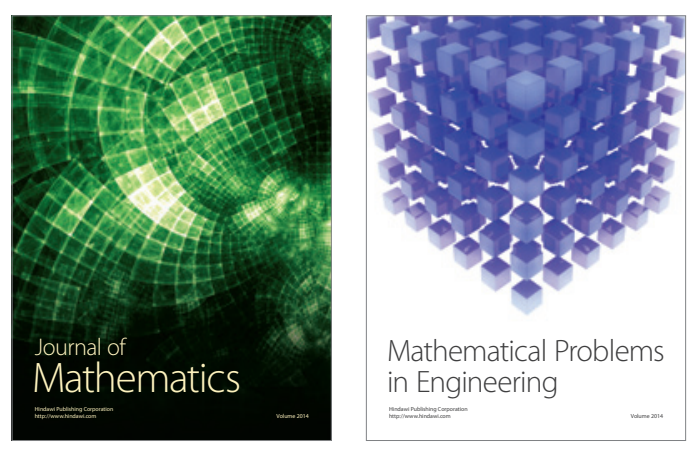

Mathematical Problems in Engineering
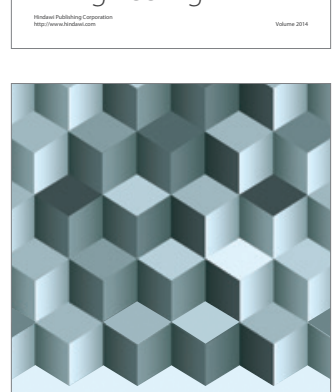

Journal of

Function Spaces
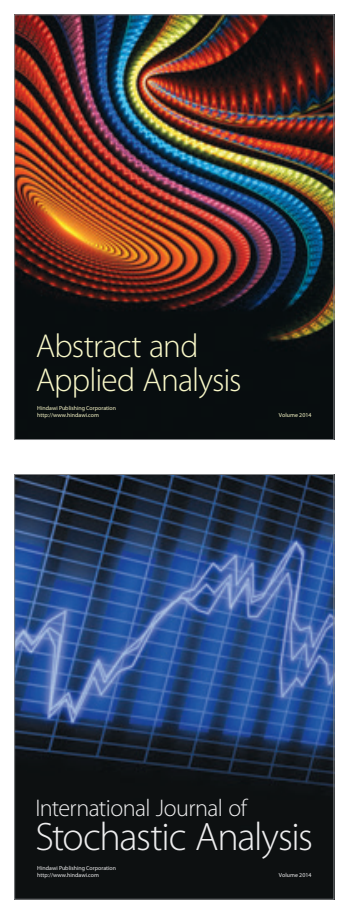

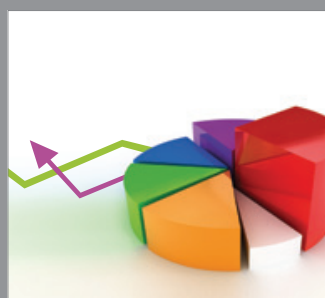

ournal of

Probability and Statistics

Promensencen
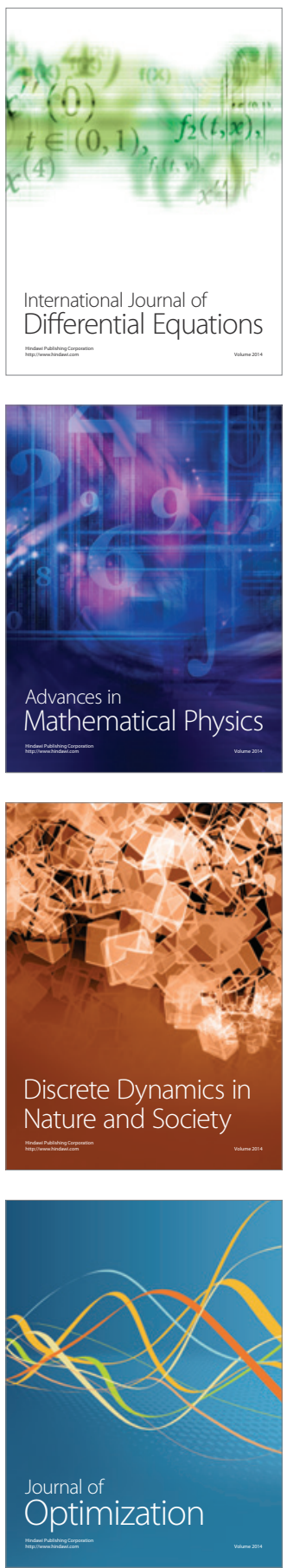